\title{
Prenatal androgen induced lean PCOS impairs mitochondria and mRNA profiles in oocytes
}

\author{
Neil R Chappell, Beth Zhou, Amy K Schutt, William E Gibbons and Chellakkan S Blesson \\ Reproductive Endocrinology and Infertility Division, Department of Obstetrics and Gynecology, Baylor College of Medicine and Family Fertility Center, \\ Texas Children's Hospital, Houston, Texas, USA
}

Correspondence should be addressed to C S Blesson: selvanes@bcm.edu

\begin{abstract}
Polycystic ovary syndrome (PCOS) is the most common ovulatory defect in women. Although most PCOS patients are obese, a subset of PCOS women are lean but show similar risks for adverse fertility outcomes. A lean PCOS mouse model was created using prenatal androgen administration. This developmentally programmed mouse model was used for this study. Our objective was to investigate if mitochondrial structure and functions were compromised in oocytes obtained from lean PCOS mouse. The lean PCOS mouse model was validated by performing glucose tolerance test, $\mathrm{HbA} 1 \mathrm{c}$ levels, body weight and estrous cycle analyses. Oocytes were isolated and were used to investigate inner mitochondrial membrane potential, oxidative stress, lipid peroxidation, ATP production, mtDNA copy number, transcript abundance and electron microscopy. Our results demonstrate that lean PCOS mice have similar weight to that of the controls but exhibit glucose intolerance and hyperinsulinemia along with dysregulated estrus cycle. Analysis of their oocytes show impaired inner mitochondrial membrane function, elevated reactive oxygen species (ROS) and increased RNA transcript abundance. Electron microscopy of the oocytes showed impaired mitochondrial ultrastructure. In conclusion, the lean PCOS mouse model shows a decreased oocyte quality related to impaired mitochondrial ultrastructure and function.
\end{abstract} Key Words

- oocytes

- glucose intolerance

\section{Introduction}

Polycystic ovary syndrome (PCOS) is the most common ovulatory disorder in the world, affecting $5-10 \%$ of women, translating to $\sim 100$ million women worldwide (1). PCOS is associated with increased obstetric risks including preeclampsia, gestational diabetes, preterm delivery and higher infant mortality (2). PCOS patients have higher miscarriage rates $(3,4)$, higher risk of ovarian hyperstimulation (OHSS) and increased risks in offspring born after assisted reproduction technology (ART) (5). Further, there are longstanding implications in chronic disease states such as hypertension, diabetes, depression, stroke and some cancers (2).

PCOS is a broad and complex disease comprised of several aberrations in physiology, leading to variable phenotypes. Diagnosis of PCOS is based on the
Rotterdam criteria: polycystic ovarian morphology, irregular menses and clinical or laboratory evidence of hyperandrogenemia (6). At least two of these three criteria will qualify a patient for the diagnosis of PCOS, provided routine workup excludes other etiologies (6). Up to $80 \%$ of PCOS patients are obese, and obesity is associated with a wide range of adverse events $(7,8,9)$, many of which are closely associated with outcomes seen in PCOS literature $(10,11)$. This makes obesity the quintessential confounder in the study of PCOS. Recently, a subpopulation of PCOS patients, that is, not obese, coined 'lean PCOS', has elicited interest among researchers $(12,13,14,15,16,17)$. This group comprises $20-30 \%$ of the PCOS population and provides the opportunity to look at PCOS independent of obesity.

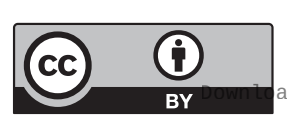

This work is licensed under a Creative Commons Attribution 4.0 International License. 
Mouse models have long served as a useful platform to study PCOS, and several models have been described, including a 'lean PCOS' mouse model via administration of androgens prenatally during the critical window of fetal oogenesis $(18,19,20,21,22,23)$. This model has since been well characterized metabolically and exhibits a PCOS-like phenotype with hyperandrogenemia, increased LH activity, irregular cyclicity and abnormal glucose/insulin tolerance while maintaining the same weight and BMI as the control population $(18,20,21)$. Despite the strong foundational data describing the metabolic components of this model, research on the reproductive system of these animals, in particular the oocyte itself, remains sparse. Prior studies have shown impaired mitochondrial function with hyperandrogenic environments in the oocyte to have negative effects on reproductive outcomes $(24,25,26,27,28,29)$. PCOS women by their nature are hyperandrogenic and thus may undergo these alterations $(30,31,32)$. Our hypothesis is that prenatal androgen administration results in a lean PCOS phenotype with impaired mitochondrial structure and function in the oocytes.

\section{Materials and methods}

All experiments were approved through the Institutional Animal Care and Use Committee of Baylor College of Medicine (AN-7156). To create the lean PCOS mouse model, 8-week-old C57/B16 female mice $(n=26)$ were mated with males of proven fertility. Copulatory plugs were visualized to confirm mating on the following morning, which was considered day 0.5. Pregnant dams were injected with $250 \mu \mathrm{g}$ of dihydrotestosterone (DHT, Sigma-Aldrich) prepared in sesame oil (Texas Lab Supply, Lubbock, TX, USA) or vehicle only on 16.5, 17.5 and 18.5 days post coitus. All male pups were culled at weaning, and female pups ( $n=30$ controls and $n=33$ DHT) were used for the experiments. One pup each from each dam was used for each experiment and sisters were not used in the same experiments. From within each litter, pups were randomly chosen for different experiments.

Pups were weighed and measured from snout to anus in millimeters at 3, 4, 8, and 12 weeks up to killing at 16 weeks to calculate their BMI. Test for estrous cyclicity was performed at 3 months of age by observing the vaginal cytology using vaginal smears as previously described (33, $34,35)$ for approximately 21 days or four consecutive cycles. Glucose tolerance test (GTT) was performed on 12-weekold mice as in previously published mouse models $(21,36)$.
Hemoglobin A1c levels were analyzed at the time of killing at 16 weeks using the A1cNow+ test kit (PTS Diagnostics, Indianapolis, IN, USA) following the manufacturer's instructions. Due to the sub-fertile nature of the animal model, in order to study the oocyte, it was necessary to perform superovulation to retrieve oocytes. The protocol is detailed in the Supplementary Materials (see section on supplementary materials given at the end of this article).

Mitochondrial function was evaluated on oocytes by single cell imaging experiments using fluorescent probes and the images were analyzed using Image $J$ software. Inner mitochondrial membrane (IMM) potential was measured using JC-1 dye (Affymetrix), reactive oxygen species (ROS) formation was measured using CellRox Green (ThermoFisher) and lipid peroxidation was measured by using BODIPY (Life Technologies). Single cell imaging of oocytes for IMM, ROS and lipid peroxidation were performed using two to five oocytes/study/group from five to eight mice.

ATP concentration was measured pooling 5 to 15 oocytes/mouse, using a luciferase assay kit (ThermoFisher). RNA transcript abundance was measured using qPCR. RNA was isolated and cDNA was amplified using three to five oocytes collected from each mouse. Genes were amplified using specific primers using cDNA library as templates. Genomic DNA was isolated and amplified to measure mitochondrial DNA copy number. During killing, ovaries from 4-month-old unstimulated mice in diestrus were collected and processed for transmission electron microscopy (TEM). The ' $n$ ' in each study corresponds to the number of mice used in each experiment. Detailed protocols utilized are described in the Supplementary Materials. Student's $t$-test, chi square and ANOVA were used where appropriate for all measurements. $P<0.05$ was considered statistically significant. The data were analyzed using GraphPad Prism version 6.0.

\section{Results}

\section{Control and PCOS mouse showed similar body weights and $\mathrm{BMI}$}

The lean PCOS mouse model exhibited similar weights when compared to controls throughout the experimental period (Fig. 1A). They did not show any difference at 16 weeks at the time of killing (control $22.48 \pm 0.47 \mathrm{~g}$ vs lean PCOS $23.67 \pm 0.29 \mathrm{~g}$ at 16 weeks, $P=0.19$ ). Further, there was no difference in BMI at 12 weeks between the two groups (control $0.25 \pm 0.007 \mathrm{~g} / \mathrm{cm}^{2}$ vs lean PCOS $0.26 \pm 0.004 \mathrm{~g} / \mathrm{cm}^{2}, P=0.22$ Fig. $\left.1 \mathrm{~B}\right)$.

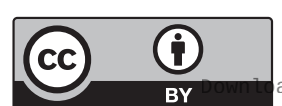

This work is licensed under a Creative Commons Attribution 4.0 International License. 

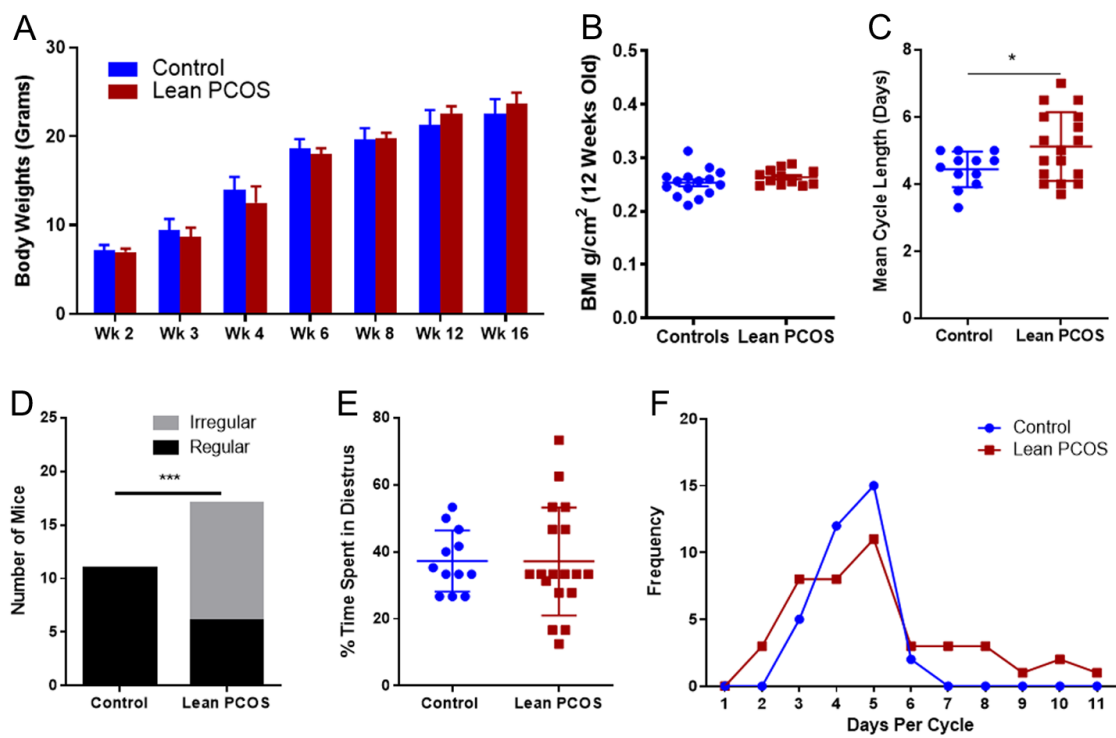

\section{Figure 1}

Lean PCOS mice displayed similar body weight (A) and BMI (B) when compared to controls. They also showed irregular cycles when compared to controls. The mean cycle length (C) in the lean PCOS group was longer, and more mice exhibited irregular cycles (D) and had more variation in the overall time spent in diestrus (E), with a wider variation in the days spent per estrus cycle (F). $(n=11-15$ in controls and 18-19 in lean PCOS, $\star p<0.05$ and $* * * p<0.001)$.

\section{Lean PCOS mice had irregular cycles}

Lean PCOS mice displayed irregular cycles when compared to controls. The mean cycle length for the lean PCOS mice was longer than that of the controls, indicating longer estrus cycles (control $4.44 \pm 0.15$ days, $n=12$, vs lean PCOS $5.12 \pm 0.25$ days, $P<0.05$; Fig. $1 C$ ). All control mice had regular estrus cycles (defined as 3-5 days per cycle); however, a significant proportion of lean PCOS mice had did not have regular cycles (control 100\% regular cycles vs lean PCOS $35 \%$ regular cycles, $n=17$, $P<0.001$; Fig. 1D). The percentage of time of spent in the diestrus was calculated for each mouse (days in diestrus/ number of days monitored). For the control population, mice spent a similar time in diestrus with a small variation (37.2\% \pm 2.6$)$, while lean PCOS mice showed significant variations $(37.1 \% \pm 3.8)$ (Fig. 1E) with days ranging from 2-10 as opposed to 3-5 days in controls (Fig. 1F). Serum obtained at 4 months was used to measure anti-Mullerian hormone (AMH) levels using standard ELISA kits, and no differences were noted between the control group and the lean PCOS group (control $68.4 \pm 7.74 \mathrm{ng} / \mathrm{mL}$ vs lean PCOS $55.08 \pm 7.8 \mathrm{ng} / \mathrm{mL}$ for $\mathrm{AMH})$.

\section{Lean PCOS mice were glucose intolerance but normal hemoglobin A1c levels}

Glucose values were consistently higher at $30 \mathrm{~min}$ (control $13.5 \pm 0.5 \mathrm{mmol} / \mathrm{L}$ vs lean PCOS $15.5 \pm 0.5 \mathrm{mmol} / \mathrm{L}$, $P<0.01$ ), $60 \mathrm{~min}$ (control $11.3 \pm 0.3 \mathrm{mmol} / \mathrm{L}$ vs lean PCOS $13.2 \pm 0.9 \mathrm{mmol} / \mathrm{L}, \quad P<0.01$ ), $120 \mathrm{~min}$ (control $8.5 \pm 0.3 \mathrm{mmol} / \mathrm{L}$ vs lean PCOS $10.2 \pm 0.2 \mathrm{mmol} / \mathrm{L}, P<0.01$ ) and $180 \mathrm{~min}$ (control $7.8 \pm 0.2 \mathrm{mmol} / \mathrm{L}$ vs lean PCOS

$9.7 \pm 0.1 \mathrm{mmol} / \mathrm{L}, \quad P<0.01$ ) during GTT (Fig. 2A). Interestingly, fasting glucose did not show any difference between the groups (Fig. 2A). The lean PCOS mice exhibited glucose intolerance when compared to controls with an increase in the GTT area under the curve (AUC), demonstrating an overall increase in glucose intolerance in the lean PCOS group when compared to the controls (Fig. $2 \mathrm{~B}$, control $1891 \pm 53.81 \mathrm{mmol} / \mathrm{L} \times 180 \mathrm{~min}$ vs lean PCOS $2121 \pm 39.56 \mathrm{mmol} / \mathrm{L} \times 180 \mathrm{~min}, P<0.01)$. The lean PCOS group showed evidence of hyperinsulinemia at the $30 \mathrm{~min}$ (Fig. 2C, control $70.134 \pm 9.68 \mathrm{pmol} / \mathrm{L}$ vs lean PCOS $114.7 \pm 14.71 \mathrm{pmol} / \mathrm{L}, P<0.05)$, with a higher

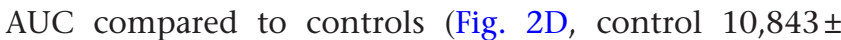
$1484 \mathrm{pmol} / \mathrm{L} \times 180 \mathrm{~min}$ vs lean PCOS 16,462 \pm $1261 \mathrm{pmol} / \mathrm{L} \times 180 \mathrm{~min}, P<0.05)$. However, there were no differences in the insulin levels in any other time point during the GTT. Further, there was no difference in hemoglobin A1c between the lean PCOS group and controls (control $4.3 \pm 0.12 \%$ vs lean PCOS $4.46 \pm 0.11 \%$ ).

\section{Inner mitochondrial membrane potential was compromised in lean PCOS oocytes}

Changes in IMM potential were assessed in live oocytes obtained from controls and lean PCOS mouse using JC-1, a dye that fluoresces red when the inner mitochondrial membrane is charged and green when depolarized. JC-1 is a marker for overall mitochondrial health and function, as this membrane potential is essential for the electron transport chain to be able to produce ATP $(29,37)$. Our analysis showed that the lean model exhibited a lower red to green ratio in oocytes collected,

https://ec.bioscientifica.com https://doi.org/10.1530/EC-19-0553 (c) 2020 The authors Published by Bioscientifica Ltd

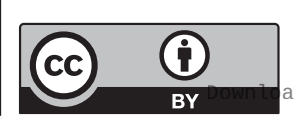

This work is licensed under a Creative Commons Attribution 4.0 International License. 
A

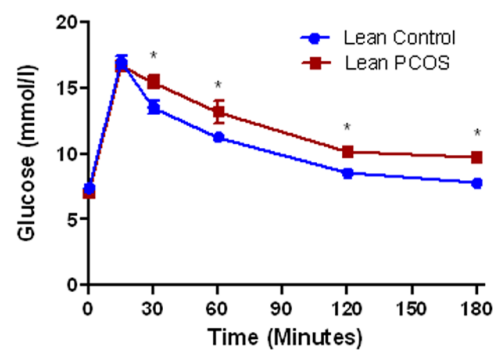

C

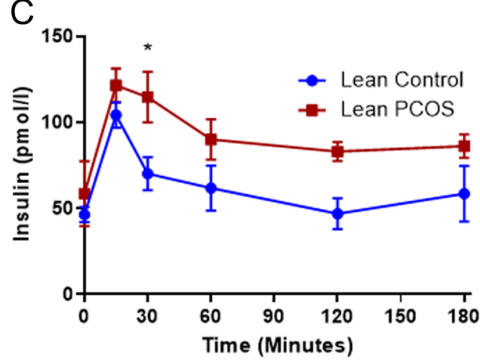

Figure 2

The lean PCOS mouse model displayed glucose intolerance (A) with increased glucose area under the curve (AUC) (B). Additionally, the lean PCOS mouse model also demonstrated hyperinsulinemia (C) with elevated insulin AUC (D). ( $n=7-8$ in controls and $n=8$ lean PCOS, $* p<0.05$ and $* * P<0.01)$

indicating a lower IMM potential (control $1.62 \pm 0.1$ vs lean PCOS 1.23 $\pm 0.09, P<0.05$; Fig. $3 \mathrm{~A}$ and $\mathrm{B})$.

\section{Oocytes from lean PCOS mice exhibited increased ROS}

ROS was measured using CellRox Green which fluoresces when oxidized by reactive oxygen species (ROS), and thus the higher the fluorescent signal measured in relative fluorescent units (RFUs), the higher the amount of ROS present. In the lean PCOS model, we observed higher RFUs compared to controls indicating a higher concentration of ROS (control 9.62 \pm 1.58 RFUs vs lean PCOS 18.46 \pm 3.25 RFUs, $P<0.05$; Fig. $4 \mathrm{~A}$ and $\mathrm{B}$ ).

\section{Lipid peroxidation was similar between the control and PCOS oocytes}

Lipid peroxidation was measured using BODIPY, a dye used to measure lipid peroxidation. Mitochondria are a major site of lipid metabolism, and peroxidation of lipids in mitochondria has been used as a marker for mitochondrial function (25). We measured mitochondrial lipid peroxidation in oocytes using a fluorescent dye, BODIPY. We observed no differences between the control and lean PCOS oocytes (control 13.8 \pm 1.51 RFUs vs lean PCOS 15.41 \pm 1.61 RFUs; Fig. 5A and B).

\section{mtDNA copy number and ATP levels showed no differences}

Mitochondrial DNA copy number and ATP production were assessed in oocytes obtained from control and lean PCOS groups. Mitochondrial DNA copy number was assessed by qPCR of a mitochondrial gene (mtCo1) compared with a somatic reference gene (tubulin). Our data show that there were no difference noted in mtDNA copy number between the groups (control $1127 \pm 707$ vs lean PCOS $1161 \pm 572$ ). ATP contents were measured from oocyte lysates and there was no
A

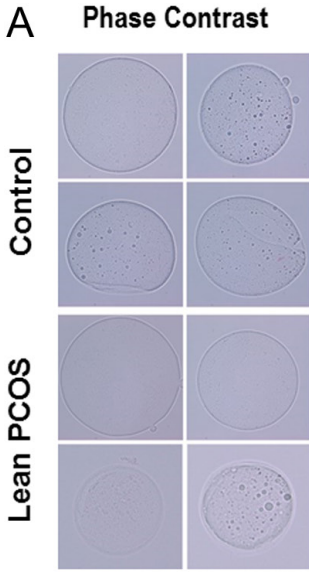

Green

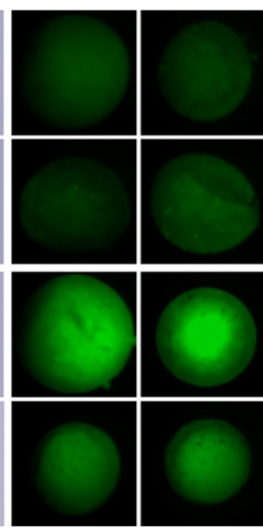

Red

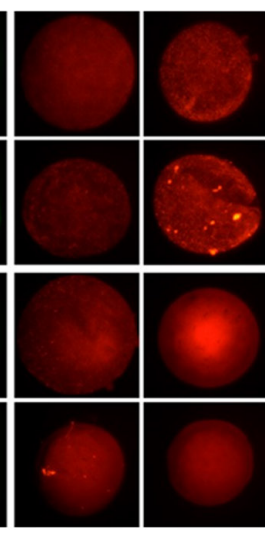

Merged

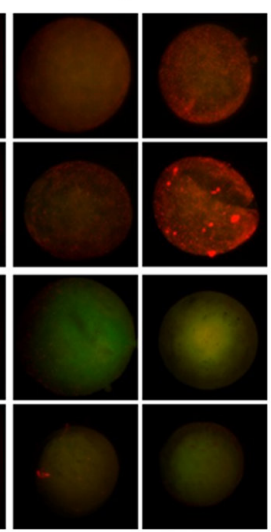

B

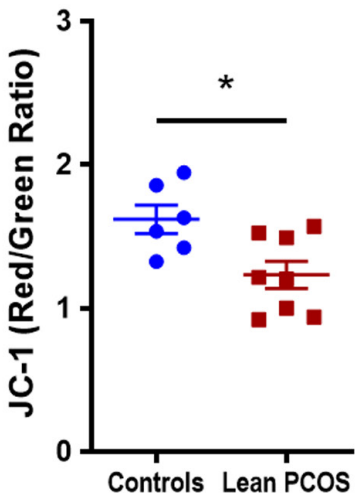

Figure 3

Pictorial representation (A) of control (upper panel) and lean PCOS (lower panel) model. Phase contrast microscopy, followed by green fluorescence, red fluorescence and merged fluorescent images using JC-1 dye at 60x magnification. The lean PCOS mice show a lower red to green ratio compared to controls (B) indicating compromised mitochondrial function. ( $n=5$ in controls and $n=8$ in lean PCOS, $* P<0.05$ ).

https://ec.bioscientifica.com

https://doi.org/10.1530/EC-19-0553 (c) 2020 The authors Published by Bioscientifica Ltd

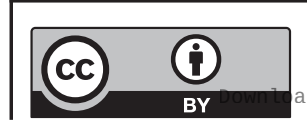

This work is licensed under a Creative Commons Attribution 4.0 International License. ded from Bioscientifica.com at $04 / 26 / 2023$ 11:37:36AM 

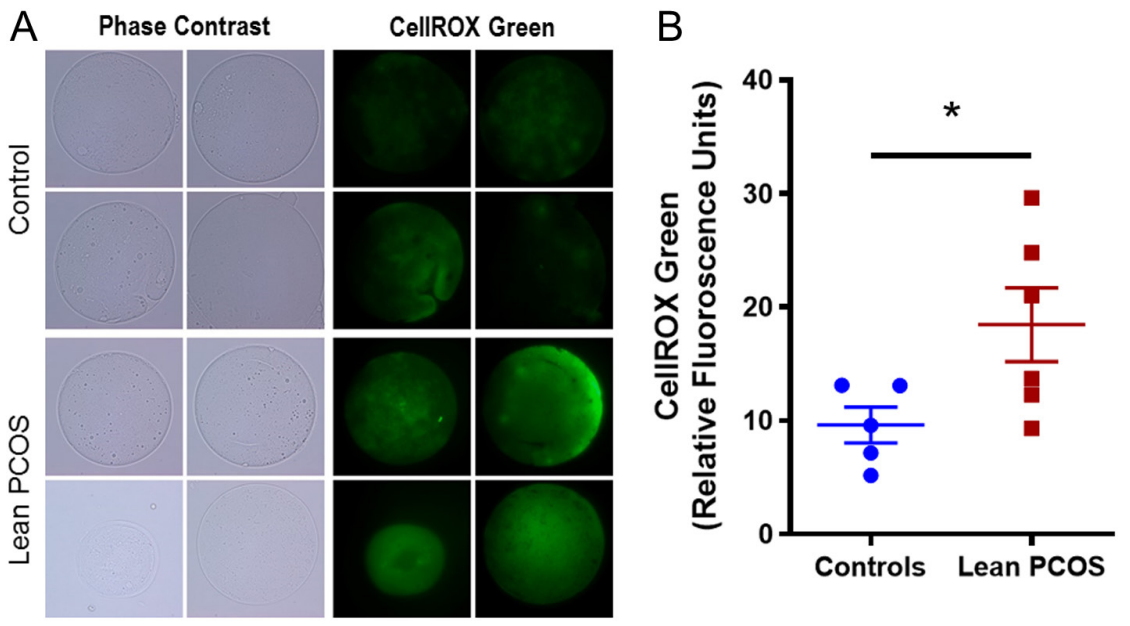

\section{Figure 4}

Pictorial representation (A) of control (upper panel) and lean PCOS (lower panel) model. Phase contrast microscopy, followed by green fluorescence using CellRox Green dye at 60x magnification. The lean PCOS mice have a higher mean RFU value indicating presence of higher reactive oxygen species concentration when compared to controls (B). $(n=5$ in controls and $n=6$ in lean PCOS, $* P<0.05)$. difference between the groups (control $21.08 \pm 4.26$ vs lean PCOS 22.15 \pm 4.66 ).

\section{Lean PCOS oocytes showed increased transcript abundance}

RNA transcript abundance was quantified using qPCR for nuclear and mitochondrial genes. Interestingly all the statistically significant differences showed increased abundance in lean PCOS group (Fig. 6). qPCR was performed for 39 genes known to be involved in PCOS and reproduction. Our results showed that five nuclear genes and seven mitochondrial genes were upregulated. Of the nuclear genes that were investigated, there was a significant increase $(P<0.05)$ in gene transcripts involved in centrosome formation (Spast), folliculogenesis (Bmp15, Zp1, Zp3 and Igfr1) and cell adhesion (Itgav6 and Dnm1). Mitochondrial genes that were upregulated $(P<0.05)$ included genes involved with complex 1 of the electron transport chain (mtNd1 and mtNd6) and complex IV
(mtCo1, mtCo2 and mtCo3). The mitochondrial genes mtNd2, mtNd3, mtNd4, mtCytb, mtAtp8 and mtRnr2 in addition to the nuclear genes Opa1, Mfn2, Ect2, Atrx, Cep70, Tacc1, Pcm1, Gdf9, Nobox, Zmic1, Kat2b, Ppp2r1a and Ppp2ca all showed a tendency to be more abundant; however, did not reach statistical significance. Mitochondrial genes mtNd4l, mtNd5, mtAtp6 and mtRnr1 as well as nuclear genes Gja1 and Sfrp4 showed similar expression in both controls and lean PCOS groups, and Xrcc1 showed a trend in lower abundance that did not reach statistical significance (Data not shown). A complete list of primers used and their details are given in the Supplementary Materials.

\section{Lean PCOS oocytes showed compromised mitochondrial ultrastructure}

TEM images of mouse oocytes showed that mitochondria in the lean PCOS group were structurally abnormal when compared to the controls. The controls had well rounded

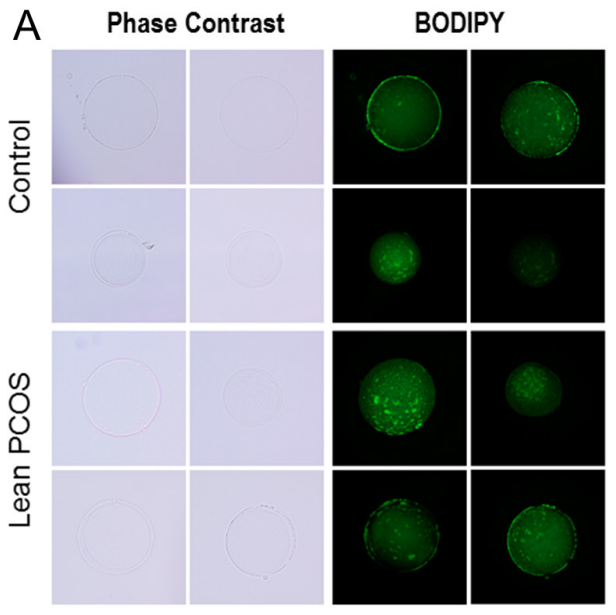

B

https://ec.bioscientifica.com https://doi.org/10.1530/EC-19-0553 (c) 2020 The authors Published by Bioscientifica Ltd

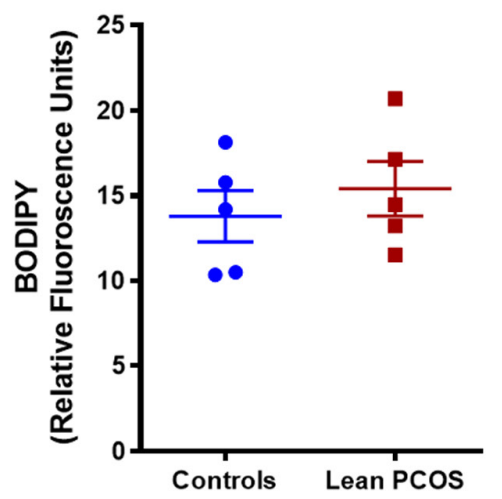

Figure 5

Pictorial representation (A) of control (upper panel) and lean PCOS (lower panel) model. Phase contrast microscopy, followed by green fluorescence using BODIPY dye at 60x magnification. The lean PCOS mice have a similar mean RFU value when compared to controls indicating no differences in lipid peroxidation between the groups (B). ( $n=5$ in controls and $n=5$ in lean PCOS). 

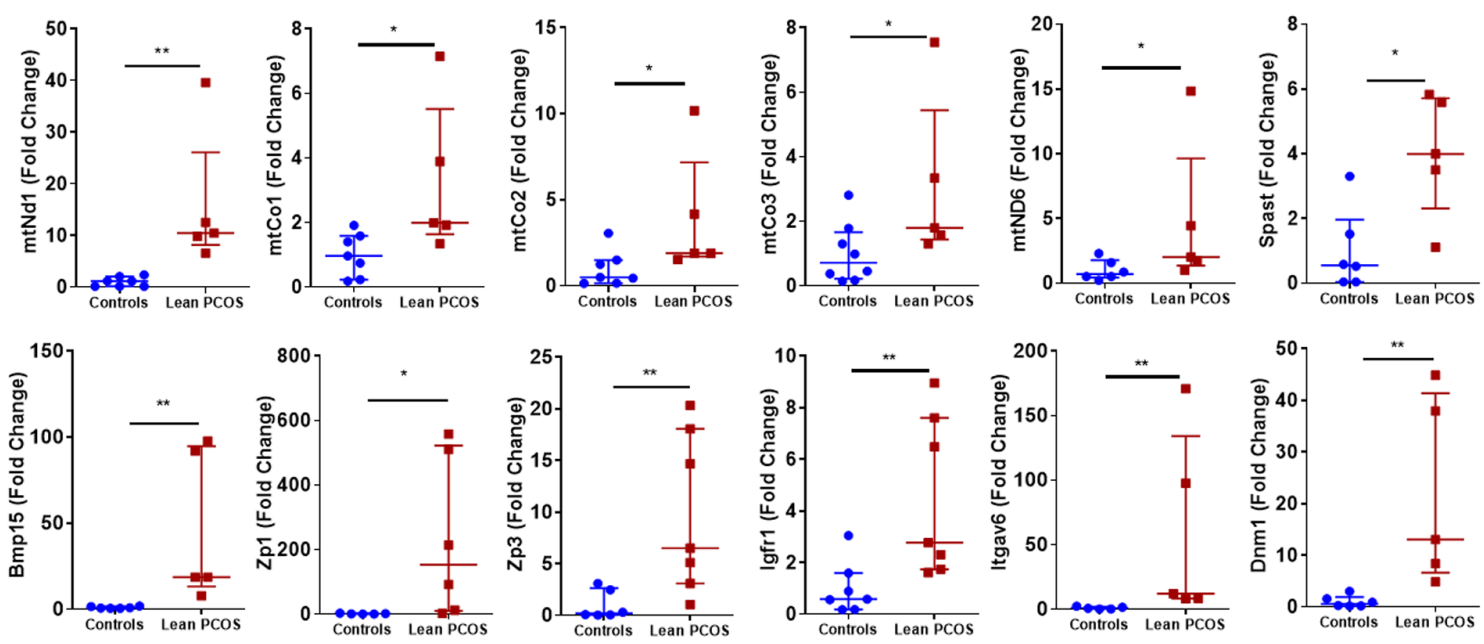

Figure 6

RNA transcript abundance in the control group vs lean PCOS mouse model. $(n=5-8, * P<0.05, * * P<0.01)$.

mitochondria with cristae at equal intervals. Further, they also had normal looking electron dense materials inside the mitochondria. Almost all the mitochondria observed had uniform shape with normal ultrastructure. Interestingly, almost all the mitochondria in oocytes from lean PCOS group showed aberrant ultrastructure with swollen cristae. Further, they were also severely vacuolated without any electron dense contents (Fig. 7A and B).

\section{Discussion}

The lean PCOS mouse model demonstrated characteristics consistent with lean PCOS patients seen in clinical practice, with metabolic abnormalities, irregular cyclicity and evidence of ovulatory dysfunction with no increase in body weight or BMI. Although we noted a slight increase (1.2 $\mathrm{g}, \sim 5 \%)$ in the body weight of lean PCOS mice at 4 months, it did not reach statistical significance $(P=0.19)$. This increase may have some possible impact, despite the lack of statistical difference. Lean PCOS mice showed glucose intolerance and hyperinsulinemia during the GTT, suggesting compromised glucose metabolism as seen in the majority of lean PCOS patients $(2,38)$. Interestingly, there was no difference in hemoglobin A1c levels compared to controls, indicating a lack of persistent hyperglycemia in these mice. Rather, they showed compensatory hyperinsulinemia indicating that higher insulin secretion was able to maintain glucose homeostasis. Such compensatory increase in insulin levels are often observed in patients with metabolic diseases; however, if unchecked will ultimately progress to worsening insulin resistance and lead to pancreatic failure (39). Earlier studies have shown that this model has elevated testosterone levels and impaired estrogen feedback (18).

To the best of our knowledge, our study presents evidence for the first time that prenatal androgen administration affects oocyte mitochondrial structure and function. The critical window of oocyte development in a mousebegins around day 16-18 of inutero fetaldevelopment (the period which the DHT was administered in this model) and continues into neonatal life. Importantly, administration of DHT after this decisive window does not carry the same effects (40). In contrast, our study clearly shows that administration of DHT during this window of fetal development affects the mitochondrial structure and function of oocytes during adulthood. The oocyte is the largest cell in the body and mitochondria are abundantly present within oocytes (27). A primordial oocyte will only have a handful of mitochondria though, throughout folliculogenesis, that number will increase to over 100,000 (27). After ovulation, this abruptly ceases, and there will be no changes in mitochondrial abundance until blastulation $(27,41)$. Therefore, a healthy amount of functional oocyte mitochondria is essential in the formation and maintenance of a strong energy reserve for the early processes of embryogenesis from fertilization through the first cell divisions (42). Thus, the implications of impaired mitochondrial function at the level of the oocyte are clear from our findings and may help to explain the adverse outcomes seen in the PCOS population.

One of the main functions of mitochondria is to generate energy in the form of ATP, which is done via 


\section{Control}

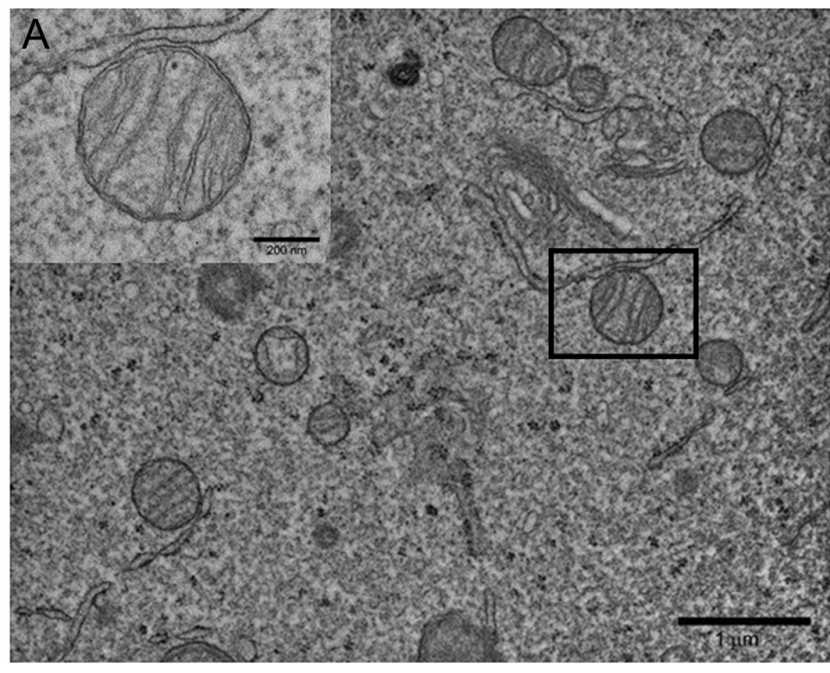

Lean PCos

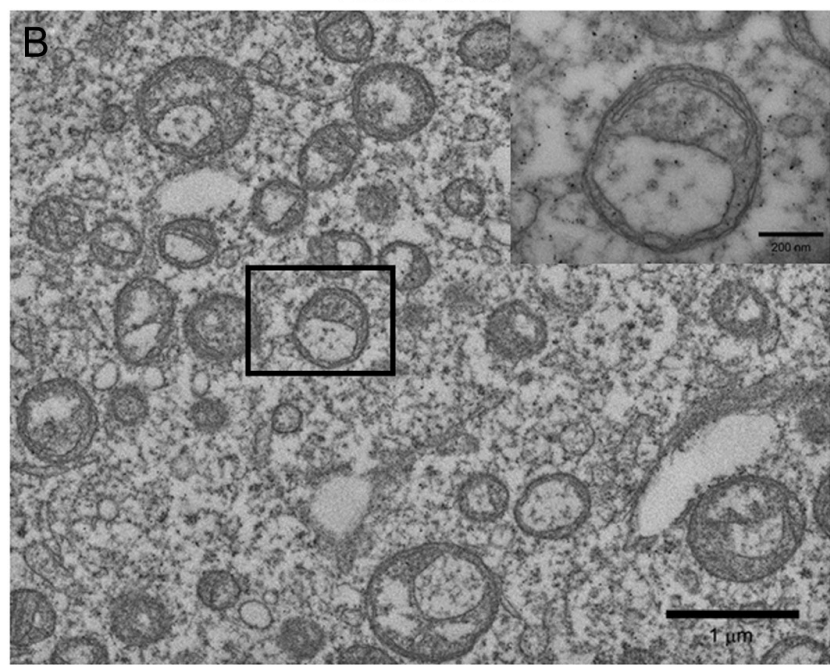

\section{Figure 7}

Representative transmission electron microscopy imaging of control $(A)$ and lean PCOS (B) mouse oocyte mitochondria. Oocytes from lean PCOS mouse show abnormal mitochondrial structure with swollen cristae ( $n=4$ in each group).

the electron transport chain (ETC) (43). The ETC is responsible for pumping protons across the IMM to generate a transmembrane gradient which is then utilized by an ATPase enzyme to create ATP (37). As the membrane potential across the IMM decreases, the ETC's function is compromised, and this has been shown to be a marker for poor mitochondrial function (37). Further, mitochondria are also integral in reactive oxygen species (ROS) formation and scavenging and regulation of apoptotic pathways (44). While a certain amount of ROS is considered normal in the oocyte, increased ROS is linked to poor reproductive outcomes via developmental arrest, apoptosis and physical
DNA damage $(29,43,45,46)$. Oocytes obtained from lean PCOS mice displayed compromised IMM potentials and increased ROS formation. Formation of ROS is tightly coupled to mitochondria; in fact, the mitochondrial genome is more susceptible to DNA damage from ROS than the nuclear genome (47). This is thought to act as a buffering mechanism, whereby excessive amounts of ROS can result in mitochondrial DNA damage which triggers apoptotic pathways before nuclear damage can accumulate to a dangerous degree (47). Mechanisms by which the mitochondria control ROS formation have been postulated to involve scavenger activity, increased antioxidant production and alterations in fission/fusion balance of mitochondrial number $(43,47,48)$.

The lack of difference in mtDNA copy number and ATP concentration despite mitochondrial damage may be due to compensatory mechanisms in PCOS oocytes. Our recent study in androgen programmed neonatal ovaries showed similar mitochondrial damage possibly due to increased oxidative stress with increased basal ATP production (49). It is possible that persistent exposure to androgens later in life may trigger alterations in mitochondrial replication that could result in differences in ATP concentration or mitochondrial copy number.

The persistence of the RNA transcripts seen in the lean PCOS mouse has exciting implications. Unlike most cells whose mRNA transcripts lasts for only a few hours (50), an oocyte transcribes mRNAs to last several days (50) from fertilization to blastulation and implantation (50). The longevity of these mRNA transcripts in this unique environment is thought to be due to changes in the adenylation of the $3^{\prime}$ tail of the mRNA, increased expression of binding proteins, inhibition of degradation pathways and through the influence of miRNAs (50). Therefore, studies of RNA expression that would typically quantify gene expression in other cell lines are more appropriately considered to be measuring quantities of RNA transcripts created prior to ovulation in the case of the oocyte (50). The majority of the genes tested in this lean model showed increased abundance of RNA transcripts in oocytes obtained from lean PCOS group, suggesting a global difference in either transcription or stabilization of these transcripts (50). The mechanism for global increase in RNA transcript DHT exposed oocytes is not known. The stabilization and prolongation of mRNA half-life can be achieved by various mechanisms (50) or there may be altered expression of miRNA transcripts, which have been reported in PCOS cohorts (51). And finally, there may be increased storage of mRNA transcripts in PCOS. In fact, studies have shown increased expression of the gene

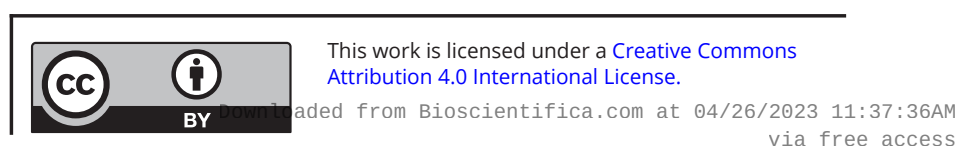


GATA6 in PCOS, which is involved in mRNA storage in the oocyte (52).

KEGG analysis of the regulated genes indicate that oxidative phosphorylation (especially in Complex I and Complex IV of the ETC) is the major pathway involved in the dysregulation. One of the main functions of the mitochondria is energy generation via the ETC, and during folliculogenesis, the number of mitochondria increase exponentially. Therefore, decreased mRNA transcript abundance of proteins involved in the ETC may indicate compromised ETC function in these oocytes. Precisely how the programming affects the growth and maturation of the oocytes and embryos that may arise from these oocytes if fertilized is not yet understood. Although the mechanisms remain unknown, a prior study in neuronal tissue of mice exposed to androgen (DHEA) has shown impairment in Complex I of mitochondrial electron transport chain (26). Other pathways involved included ovarian steroidogenesis, meiosis and signaling via the AMPK and PI3kAkt pathways. Multiple genes involved in folliculogenesis including Bmp15 and Igfr1 were also increased, highlighting the dysregulation in oocyte maturation that is seen in PCOS. Interestingly, KEGG analysis also highlighted pathways involved in Parkinson's disease and depression, the latter of which is well known to be associated with PCOS, but the role of these RNA transcripts in the oocyte with these disorders is unknown at this time (2).

Finally, as these changes in the oocyte mitochondria are transferred to the offspring, this may explain the inheritance pattern seen in the offspring of PCOS patients (53). PCOS is well known to be hereditary, though numerous studies investigating the genetic contributions to the disease have been unable to identify strong gene candidates to explain this pattern $(54,55,56)$. Other alternative explanations may include alterations in mitochondrial DNA, increased cellular stress resulting in apoptosis or perhaps via direct effects to the mitochondria itself $(26,29,42)$. Furthermore, it stands to reason that the propagation of these mitochondria from a fertilized egg to a blastocyst and ultimately a live-born offspring should result in abnormal mitochondrial function throughout the body. Recent studies in a rat PCOS model have noted mitochondrial dysfunction in the pancreas (57) and kidney (58), and human studies have now reported altered mitochondrial function in skeletal muscle (59) as well as follicular fluid and cumulus cells of PCOS patients (46).

In conclusion, our findings indicate that prenatal androgen programming affects mitochondrial function and structure in oocytes. It is likely that the prenatal administration of androgens during the window of fetal oogenesis affects the mitochondria in the fetal oocyte along with dysregulated transcript abundance.

\section{Supplementary materials}

This is linked to the online version of the paper at https://doi.org/10.1530/ EC-19-0553.

\section{Declaration of interest}

The authors declare that there is no conflict of interest that could be perceived as prejudicing the impartiality of the research reported.

\section{Funding}

This work was supported by training grants by the Department of Obstetrics and Gynecology, Baylor College of Medicine (N C and B Z) and R-01 research grant (Grant \# DK114689) for CSB from National Institutes of Health.

\section{Acknowledgements}

The authors would like to acknowledge the Department of Obstetrics and Gynecology at the Baylor College of Medicine for their support in this project. The authors would also like to acknowledge Dr Ignatia Van den Veyver and Dr JoAnne Richards for their input as well as the Baylor College of Medicine Integrative Microscopy Core and the Animal Facility for their assistance.

\section{References}

1 Goodarzi MO, Dumesic DA, Chazenbalk G \& Azziz R. Polycystic ovary syndrome: etiology, pathogenesis and diagnosis. Nature Reviews: Endocrinology 20117 219-231. (https://doi.org/10.1038/ nrendo.2010.217)

2 Palomba S, Santagni S, Falbo A \& La Sala GB. Complications and challenges associated with polycystic ovary syndrome: current perspectives. International Journal of Women's Health 20157 745-763. (https://doi.org/10.2147/IJWH.S70314)

3 Liu L, Tong X, Jiang L, Li TC, Zhou F \& Zhang S. A comparison of the miscarriage rate between women with and without polycystic ovarian syndrome undergoing IVF treatment. European Journal of Obstetrics, Gynecology, and Reproductive Biology 2014176 178-182. (https://doi.org/10.1016/j.ejogrb.2014.02.041)

4 Luo L, Gu F, Jie H, Ding C, Zhao Q, Wang Q \& Zhou C. Early miscarriage rate in lean polycystic ovary syndrome women after euploid embryo transfer - a matched-pair study. Reproductive Biomedicine Online 201735 576-582. (https://doi.org/10.1016/j. rbmo.2017.07.010)

5 Grigorescu V, Zhang Y, Kissin DM, Sauber-Schatz E, Sunderam M, Kirby RS, Diop H, McKane P \& Jamieson DJ. Maternal characteristics and pregnancy outcomes after assisted reproductive technology by infertility diagnosis: ovulatory dysfunction versus tubal obstruction. Fertility and Sterility 2014101 1019-1025. (https://doi.org/10.1016/j. fertnstert.2013.12.030)

6 Rotterdam ESHRE/ASRM-Sponsored PCOS Consensus Workshop Group. Revised 2003 consensus on diagnostic criteria and long-term health risks related to polycystic ovary syndrome. Fertility and Sterility 200481 19-25. (https://doi.org/10.1016/j.fertnstert.2003.10.004)

7 Broughton DE \& Moley KH. Obesity and female infertility: potential mediators of obesity's impact. Fertility and Sterility 2017107 840-847. (https://doi.org/10.1016/j.fertnstert.2017.01.017)

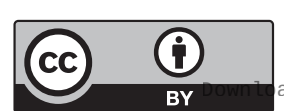


8 Practice Committee of the American Society for Reproductive Medicine. Obesity and reproduction: a committee opinion. Fertility and Sterility 2015104 1116-1126. (https://doi.org/10.1016/j. fertnstert.2015.08.018)

9 Shah DK, Missmer SA, Berry KF, Racowsky C \& Ginsburg ES. Effect of obesity on oocyte and embryo quality in women undergoing in vitro fertilization. Obstetrics and Gynecology 2011118 63-70. (https://doi. org/10.1097/AOG.0b013e31821fd360)

10 Bailey AP, Hawkins LK, Missmer SA, Correia KF \& Yanushpolsky EH. Effect of body mass index on in vitro fertilization outcomes in women with polycystic ovary syndrome. American Journal of Obstetrics and Gynecology 2014211 163.e1-163.e6. (https://doi. org/10.1016/j.ajog.2014.03.035)

11 Cardozo E, Pavone ME \& Hirshfeld-Cytron JE. Metabolic syndrome and oocyte quality. Trends in Endocrinology and Metabolism 201122 103-109. (https://doi.org/10.1016/j.tem.2010.12.002)

12 Altuntas Y, Bilir M, Ucak S \& Gundogdu S. Reactive hypoglycemia in lean young women with PCOS and correlations with insulin sensitivity and with beta cell function. European Journal of Obstetrics, Gynecology, and Reproductive Biology 2005119 198-205. (https://doi. org/10.1016/j.ejogrb.2004.07.038

13 Caglar GS, Kahyaoglu I, Pabuccu R, Demirtas S \& Seker R. AntiMullerian hormone and insulin resistance in classic phenotype lean PCOS. Archives of Gynecology and Obstetrics 2013288 905-910. (https://doi.org/10.1007/s00404-013-2833-9)

14 Mario FM, do Amarante F, Toscani MK \& Spritzer PM. Lean muscle mass in classic or ovulatory PCOS: association with central obesity and insulin resistance. Experimental and Clinical Endocrinology and Diabetes 2012120 511-516. (https://doi. org/10.1055/s-0032-1309006)

15 Shumak SL. Even lean women with PCOS are insulin resistant. BMJ 2009338 b954. (https://doi.org/10.1136/bmj.b954)

16 Kriseman M, Mills C, Kovanci E, Sangi-Haghpeykar H \& Gibbons W. AntiMullerian hormone levels are inversely associated with body mass index (BMI) in women with polycystic ovary syndrome. Journal of Assisted Reproduction and Genetics 201532 1313-1316. (https://doi. org/10.1007/s10815-015-0540-0)

17 McCormick B, Thomas M, Maxwell R, Williams D \& Aubuchon M. Effects of polycystic ovarian syndrome on in vitro fertilizationembryo transfer outcomes are influenced by body mass index. Fertility and Sterility 200890 2304-2309. (https://doi.org/10.1016/j. fertnstert.2007.10.077)

18 Moore AM, Prescott M \& Campbell RE. Estradiol negative and positive feedback in a prenatal androgen-induced mouse model of polycystic ovarian syndrome. Endocrinology 2013154 796-806. (https://doi.org/10.1210/en.2012-1954)

19 Moore AM, Prescott M, Marshall CJ, Yip SH \& Campbell RE. Enhancement of a robust arcuate GABAergic input to gonadotropinreleasing hormone neurons in a model of polycystic ovarian syndrome. PNAS 2015112 596-601. (https://doi.org/10.1073/ pnas.1415038112)

20 Roland AV \& Moenter SM. Reproductive neuroendocrine dysfunction in polycystic ovary syndrome: insight from animal models. Frontiers in Neuroendocrinology 201435 494-511. (https://doi.org/10.1016/j. yfrne.2014.04.002)

21 Roland AV, Nunemaker CS, Keller SR \& Moenter SM. Prenatal androgen exposure programs metabolic dysfunction in female mice. Journal of Endocrinology 2010207 213-223. (https://doi.org/10.1677/JOE-10-0217)

22 Sullivan SD \& Moenter SM. Prenatal androgens alter GABAergic drive to gonadotropin-releasing hormone neurons: implications for a common fertility disorder. PNAS 2004101 7129-7134. (https://doi. org/10.1073/pnas.0308058101)

23 Caldwell AS, Middleton LJ, Jimenez M, Desai R, McMahon AC, Allan CM, Handelsman DJ \& Walters KA. Characterization of reproductive, metabolic, and endocrine features of polycystic ovary syndrome in female hyperandrogenic mouse models. Endocrinology 2014155 3146-3159. (https://doi.org/10.1210/en.2014-1196)
24 Fragouli E, Spath K, Alfarawati S, Kaper F, Craig A, Michel CE, Kokocinski F, Cohen J, Munne S \& Wells D. Altered levels of mitochondrial DNA are associated with female age, aneuploidy, and provide an independent measure of embryonic implantation potential. PLoS Genetics 201511 e1005241. (https://doi.org/10.1371/ journal.pgen.1005241)

25 Huang Y, Yu Y, Gao J, Li R, Zhang C, Zhao H, Zhao Y \& Qiao J. Impaired oocyte quality induced by dehydroepiandrosterone is partially rescued by metformin treatment. PLOS ONE $2015 \mathbf{1 0}$ e0122370. (https://doi.org/10.1371/journal.pone.0122370)

26 Safiulina D, Peet N, Seppet E, Zharkovsky A \& Kaasik A. Dehydroepiandrosterone inhibits complex I of the mitochondrial respiratory chain and is neurotoxic in vitro and in vivo at high concentrations. Toxicological Sciences 200693 348-356. (https://doi. org/10.1093/toxsci/kfl064)

27 St John JC, Facucho-Oliveira J, Jiang Y, Kelly R \& Salah R. Mitochondrial DNA transmission, replication and inheritance: a journey from the gamete through the embryo and into offspring and embryonic stem cells. Human Reproduction Update 201016 488-509. (https://doi.org/10.1093/humupd/dmq002)

28 Tarumi W, Tsukamoto S, Okutsu Y, Takahashi N, Horiuchi T, Itoh MT \& Ishizuka B. Androstenedione induces abnormalities in morphology and function of developing oocytes, which impairs oocyte meiotic competence. Fertility and Sterility 201297 469-476. (https://doi. org/10.1016/j.fertnstert.2011.11.040)

29 Van Blerkom J. Mitochondrial function in the human oocyte and embryo and their role in developmental competence. Mitochondrion 201111 797-813. (https://doi.org/10.1016/j.mito.2010.09.012)

30 Daan NM, Koster MP, Steegers-Theunissen RP, Eijkemans MJ \& Fauser BC. Endocrine and cardiometabolic cord blood characteristics of offspring born to mothers with and without polycystic ovary syndrome. Fertility and Sterility $2017 \mathbf{1 0 7}$ 261.e3-268.e3. (https://doi. org/10.1016/j.fertnstert.2016.09.042)

31 Dumesic DA, Schramm RD, Peterson E, Paprocki AM, Zhou R \& Abbott DH. Impaired developmental competence of oocytes in adult prenatally androgenized female rhesus monkeys undergoing gonadotropin stimulation for in vitro fertilization. Journal of Clinical Endocrinology and Metabolism 200287 1111-1119. (https://doi. org/10.1210/jcem.87.3.8287)

32 Xita N \& Tsatsoulis A. Review: fetal programming of polycystic ovary syndrome by androgen excess: evidence from experimental, clinical, and genetic association studies. Journal of Clinical Endocrinology and Metabolism 200691 1660-1666. (https://doi.org/10.1210/jc.20052757)

33 Byers SL, Wiles MV, Dunn SL \& Taft RA. Mouse estrous cycle identification tool and images. PLOS ONE 20127 e35538. (https:// doi.org/10.1371/journal.pone.0035538)

34 Nelson JF, Felicio LS, Randall PK, Sims C \& Finch CE. A longitudinal study of estrous cyclicity in aging C57BL/6J mice: I. Cycle frequency, length and vaginal cytology. Biology of Reproduction 198227 327-339. (https://doi.org/10.1095/biolreprod27.2.327)

35 Caligioni CS. Assessing reproductive status/stages in mice. Current Protocols in Neuroscience 2009 Appendix 4 Appendix 4I. (https://doi. org/10.1002/0471142301.nsa04is48)

36 Andrikopoulos S, Blair AR, Deluca N, Fam BC \& Proietto J. Evaluating the glucose tolerance test in mice. American Journal of Physiology: Endocrinology and Metabolism 2008295 E1323-E1332. (https://doi. org/10.1152/ajpendo.90617.2008)

37 Seidler EA \& Moley KH. Metabolic determinants of mitochondrial function in oocytes. Seminars in Reproductive Medicine 201533 396-400. (https://doi.org/10.1055/s-0035-1567822)

38 Norman RJ, Dewailly D, Legro RS \& Hickey TE. Polycystic ovary syndrome. Lancet 2007370 685-697. (https://doi.org/10.1016/ S0140-6736(07)61345-2)

39 Morciano A, Romani F, Sagnella F, Scarinci E, Palla C, Moro F, Tropea A, Policola C, Della Casa S, Guido M, et al. Assessment of insulin resistance in lean women with polycystic ovary syndrome.

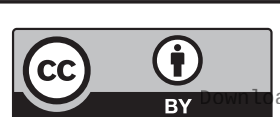

This work is licensed under a Creative Commons Attribution 4.0 International License. ded from Bioscientifica com at 04/26/2023 11:37:36 AM 
Fertility and Sterility 2014102 250-256.e3. (https://doi.org/10.1016/j. fertnstert.2014.04.004)

40 Walters KA. Role of androgens in normal and pathological ovarian function. Reproduction 2015149 R193-R218. (https://doi. org/10.1530/REP-14-0517)

41 Meldrum DR, Casper RF, Diez-Juan A, Simon C, Domar AD \& Frydman R. Aging and the environment affect gamete and embryo potential: can we intervene? Fertility and Sterility 2016105 548-559. (https://doi.org/10.1016/j.fertnstert.2016.01.013)

42 Babayev E \& Seli E. Oocyte mitochondrial function and reproduction. Current Opinion in Obstetrics and Gynecology 201527 175-181. (https://doi.org/10.1097/GCO.0000000000000164)

43 Takahashi M. Oxidative stress and redox regulation on in vitro development of mammalian embryos. Journal of Reproduction and Development 201258 1-9. (https://doi.org/10.1262/jrd.11-138n)

44 Vasconsuelo A, Pronsato L, Ronda AC, Boland R \& Milanesi L. Role of 17beta-estradiol and testosterone in apoptosis. Steroids $20117 \mathbf{6}$ 1223-1231. (https://doi.org/10.1016/j.steroids.2011.08.001)

45 Treidel LA, Whitley BN, Benowitz-Fredericks ZM \& Haussmann MF. Prenatal exposure to testosterone impairs oxidative damage repair efficiency in the domestic chicken (Gallus gallus). Biology Letters 2013 9 20130684. (https://doi.org/10.1098/rsbl.2013.0684)

46 Zhao H, Zhao Y, Li T, Li M, Li J, Li R, Liu P, Yu Y \& Qiao J. Metabolism alteration in follicular niche: the nexus among intermediary metabolism, mitochondrial function, and classic polycystic ovary syndrome. Free Radical Biology and Medicine 201586 295-307. (https://doi.org/10.1016/j.freeradbiomed.2015.05.013)

47 Vasconsuelo A, Milanesi L \& Boland R. Actions of 17beta-estradiol and testosterone in the mitochondria and their implications in aging. Ageing Research Reviews 201312 907-917. (https://doi. org/10.1016/j.arr.2013.09.001)

48 Karamohamed S \& Guidotti G. Bioluminometric method for realtime detection of ATPase activity. BioTechniques $200131420-425$. (https://doi.org/10.2144/01312rr04)

49 Barsky M, Yang L, Hosseinzadeh P, Dunn JC, Gibbons WE \& Blesson CS. Fetal programming of polycystic ovary syndrome (PCOS): in utero androgen exposure alters murine prenatal ovarian mitochondrial structure and function. In Reproductive Sciences, pp 282A. Thousand Oaks, CA, USA: Sage Publications, 2019.

50 Sirard MA. Factors affecting oocyte and embryo transcriptomes. Reproduction in Domestic Animals 201247 (Supplement 4) 148-155. (https://doi.org/10.1111/j.1439-0531.2012.02069.x)
51 Sorensen AE, Udesen PB, Wissing ML, Englund ALM \& Dalgaard LT. MicroRNAs related to androgen metabolism and polycystic ovary syndrome. Chemico-Biological Interactions 2016259 8-16. (https:// doi.org/10.1016/j.cbi.2016.06.008)

52 Wood JR, Ho CK, Nelson-Degrave VL, McAllister JM \& Strauss 3rd JF. The molecular signature of polycystic ovary syndrome (PCOS) theca cells defined by gene expression profiling. Journal of Reproductive Immunology 200463 51-60. (https://doi.org/10.1016/j. jri.2004.01.010)

53 Pan JX, Zhang JY, Ke ZH, Wang FF, Barry JA, Hardiman PJ \& Qu F. Androgens as double-edged swords: induction and suppression of follicular development. Hormones 201514 190-200. (https://doi. org/10.14310/horm.2002.1580)

54 Hayes MG, Urbanek M, Ehrmann DA, Armstrong LL, Lee JY, Sisk R, Karaderi T, Barber TM, McCarthy MI, Franks S, et al. Genome-wide association of polycystic ovary syndrome implicates alterations in gonadotropin secretion in European ancestry populations. Nature Communications 20156 7502. (https://doi.org/10.1038/ ncomms8502)

55 Liu H, Zhao H \& Chen ZJ. Genome-wide association studies for polycystic ovary syndrome. Seminars in Reproductive Medicine 201634 224-229. (https://doi.org/10.1055/s-0036-1585403)

56 Zhao H, Lv Y, Li L \& Chen ZJ. Genetic studies on polycystic ovary syndrome. Best Practice and Research: Clinical Obstetrics and Gynaecology 201637 56-65. (https://doi.org/10.1016/j. bpobgyn.2016.04.002)

57 Wang H, Wang X, Zhu Y, Chen F, Sun Y \& Han X. Increased androgen levels in rats impair glucose-stimulated insulin secretion through disruption of pancreatic beta cell mitochondrial function. Journal of Steroid Biochemistry and Molecular Biology 2015154 254-266. (https://doi.org/10.1016/j.jsbmb.2015.09.003)

58 Selen ES, Bolandnazar Z, Tonelli M, Butz DE, Haviland JA, Porter WP $\&$ Assadi-Porter FM. NMR metabolomics show evidence for mitochondrial oxidative stress in a mouse model of polycystic ovary syndrome. Journal of Proteome Research 201514 3284-3291. (https:// doi.org/10.1021/acs.jproteome.5b00307)

59 Cree-Green M, Rahat H, Newcomer BR, Bergman BC, Brown MS, Coe GV, Newnes L, Garcia-Reyes Y, Bacon S, Thurston JE, et al. Insulin resistance, hyperinsulinemia, and mitochondria dysfunction in nonobese girls with polycystic ovarian syndrome. Journal of the Endocrine Society 20171 931-944. (https://doi.org/10.1210/js.201700192)

Received in final form 23 January 2020

Accepted 25 January 2020

Accepted Manuscript published online 26 January 2020 https://ec.bioscientifica.com https://doi.org/10.1530/EC-19-0553 (c) 2020 The authors Published by Bioscientifica Ltd

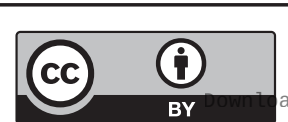

This work is licensed under a Creative Commons Attribution 4.0 International License. ded from Bioscientifica.com at 04/26/2023 11:37:36AM 